Substance and Seduction 
THE WILLIAM Q B BTTYE NOWLIN SERIES in Art, History, and Culture of the Western Hemisphere 


\section{Substance and Seduction}

INGESTED COMMODITIES IN

EARLY MODERN MESOAMERICA

Edited by Stacey Schwartzkopf and Katbryn E. Sampeck 
Copyright (C) 2017 by the University of Texas Press

All rights reserved

Printed in the United States of America

First edition, 2017

Requests for permission to reproduce material from this work should be sent to:

Permissions

University of Texas Press

P.O. Box 7819

Austin, TX 78713-7819

utpress.utexas.edu/rp-form

@ $@$ The paper used in this book meets the minimum requirements of ANSI/NISO Z39.48-1992 (R1997) (Permanence of Paper).

LIBRARY OF CONGRESS CATALOGING-IN-PUBLICATION DATA

Names: Schwartzkopf, Stacey, editor.|Sampeck, Kathryn E., editor.

Title: Substance and seduction : ingested commodities in early modern

Mesoamerica / edited by Stacey Schwartzkopf and Kathryn E.

Sampeck.

Description: First edition. | Austin : University of Texas Press, 2017. |

Includes bibliographical references and index.

Identifiers: LCCN 2017012667| ISBN 978-1-4773-1386-2 (cloth : alk. paper)|

ISBN 978-1-4773-1387-9 (pbk. : alk. paper) | ISBN 978-1-4773-1388-6

(library e-book) | ISBN 978-1-4773-1389-3 (nonlibrary e-book)

Subjects: LCsH: Mayas-Food. | Mayas-Substance use-Social aspects. |

Central America-Social life and customs. | Food consumption-

Social aspects-Central America. | Central America-Colonization. |

Colonization-Social aspects-Central America.| Consumption

(Economics)-Central America.| Hallucinogenic drugs and religious

experience-Central America. | Ingestion-Central America-

Psychological aspects-History.

Classification: LCC F1435.3.F7 S83 2017 | DDC 972.81-dc23

LC record available at https://lccn.loc.gov/2017012667

doi: $10.7560 / 313862$ 